\title{
Analysis on Creative Structure Design of Women's Dress Based on Dart Transfer
}

\author{
Jing An \\ Huanghe Science and Technology College \\ Zhengzhou, China
}

\begin{abstract}
As a bridge connecting style design and production process, the garment structural design itself also contains many creative elements and thinking methods. This paper discusses garment creative structure design from the perspective of dart transfer, to analyze the idea and method of structural creativity through the perspectives such as dart transfer, dividing line design, pleat design, three-dimensional shape and comprehensive elements application, thus to ultimately decode the creative structure design of women's dress.
\end{abstract}

Keywords—design of women's dress; dart transfer; creative structure

\section{INTRODUCTION}

Modern garment production mainly consists of three links, namely, garment style design, structural design and production process. Garment style design mainly solves the problems relating to garment design orientation, profile and detail, fabric and color and other visual aesthetic issues; garment structural design is the process changing design sketch into plane pattern, and finally complete the sewing of clothes through production process. From an overall perspective, the latter two processes belong to the engineering technology category, which is far away from the creative design. But with the international development trend in garment industry in recent years, garment creative design has penetrated into all aspects. For example, in the developed countries such as the United Kingdom and Japan, the garment structure designer is a special position. He opens up the specific division of labor of style design and engineering drawing, and launches design from internal technology elements such as garment function, structure drawing and production process to external aesthetic, which has realized the design ideas of garment from inside out. However, in China, garment industry still remains a clear division of design and engineering positions, which is conducive to mass production, production costs reduction, but hindered the launch of garment creative design in many areas. For example, in the clothing enterprises, many designers' creative ideas cannot be achieved by pattern maker. So the pattern maker starts to guide designers to modify the design draft, which often wipes out the designer's original design, and such situation is quite common. Therefore, connecting garment style design and production process, structural design plays a decisive role.

\section{GARMENT CREATIVE STRUCTURAL DESIGN AND THE MEANING OF DART}

First of all, let us first learn about what is auspicious pattern. During the long years, our ancestors created a lot of patterns yearning for freedom, pursuing better life with auspicious meaning. These patterns skillfully use characters, animals, birds and flowers, the sun, the moon and stars, lightning and storm, words, to create the art form combining graphic and auspicious meaning perfectly with the myths and legends as well as folk proverbs as the theme by virtue of analogy, pun, and homophonic, symbolic and other techniques. We call such patterns with historical origin, rich folk characteristics, and auspicious expecting as Chinese traditional auspicious patterns. According to the application carrier of auspicious patterns, they can be divided into architectural decoration patterns, furniture decoration, printing and dyeing embroidery patterns, porcelain patterns, lacquer patterns and so on. According to the theme of auspicious patterns, they can be divided into characters, animals, plants, words, geometric patterns and other combinations. Garment structural design, or pattern design, is the plane unfolding process of various parts based on garment design sketch or style drawings. This process is often calculated and drawn based on human structure and size. But with the arrival of the era of personalized garment design, now it also incorporates more creative ideas and methods, becoming an important means of garment styling design, which can be called the garment creative structure design. Of course, the garment structural design is inseparable from the understanding and use of dart, because the human body is not a simple cylindrical or spherical shape, but a complex three-dimensional, the difference between chest and waist determines the complex changes in garment structure. In order to make the plane-like cloth correspond to the complexity of human body surface, it is a must to start from the treatment method of garment structure.

Dart, as the name implies, is omission, meaning to save, which refers to transfer the cloth from plane to a threedimensional structure that conforms to human body structure by cutting off extra cloth from the plane cloth, and the volume that has been cut off is called as "dart volume", the edges after seaming is called as "dart" (Fig. 1). After dart processing, flat fabrics will show a conical or round table and other threedimensional effect, so that the piece of clothes or garment will fit human body to a high degree (Fig. 2). For example, after the bust dart and waist dart are sewed, the surface is conical, in 
paper pattern, it usually presents as V-shape or "jujube core" shape. It is the use of dart that makes clothing fit human body from the loose state.

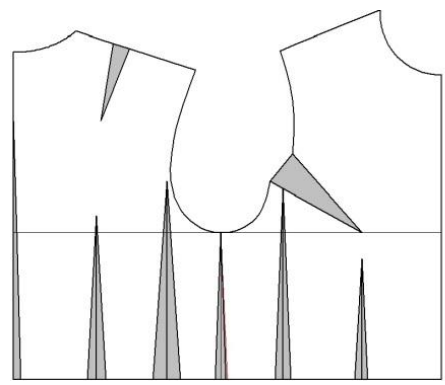

Fig. 1. Women's Garment Prototype with Japanese Culture
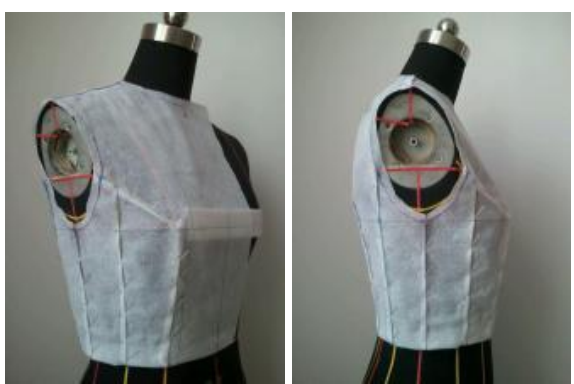

Fig. 2. Women's Garment Prototype in kind with Japanese Culture

\section{DART ClASSIFICATION AND TRANSFER METHOD IN Clothing}

Female body surface is composed of multiple complex surfaces, so the darts spread all over various parts of the garment. Darts at different parts have different locations and appearances, which can be divided into two categories:

First, according to the location of darts, those located at the breast convex are called breast darts, those located in the waist are called waist darts, and those located at back shoulder blade are called as shoulder blade dart. Around the body bumps, while maintaining the garment fit degree unchanged, a variety of new darts will be formed if the dart's location is changed. For example, the dart at collar is called collar dart, the dart at front fly is called seam dart, and the dart at armpit is known as the armpit dart (Fig. 3, Fig. 4).

Second, divide according to the shape of darts. The dart having a shape similar to the shape of a nail, with the upper part quite parallel, and the lower part as a sharp shape, is called nail dart, which is commonly used to shape the surfaces of complex shape at shoulder and chest, such as shoulder dart, collar dart and other darts. The dart having a shape similar to cone is called cone dart, which is commonly used in the production of conical surface, such as the waist dart, sleeve elbow dart. One end of the dart is sharp shape, the other end is non-fixed shape, or both ends are non-fixed shapes, then it is called known as dart tucks, which is a decorative and functional dart. If both ends of the dart is sharp, and the middle is wide, then it is called as "olive dart" or "jujube dart", which is commonly used in the waist dart of upper garment. If the dart has an arc shape, and if the dart becomes smaller gradually from top to bottom, or the upper is parallel, the lower part goes into a sharp shape, then it is call arc dart, having both decorative and functional role. The simple plane becomes into complex surface by adding a dart, which has constituted the diversified garment fitting human body.
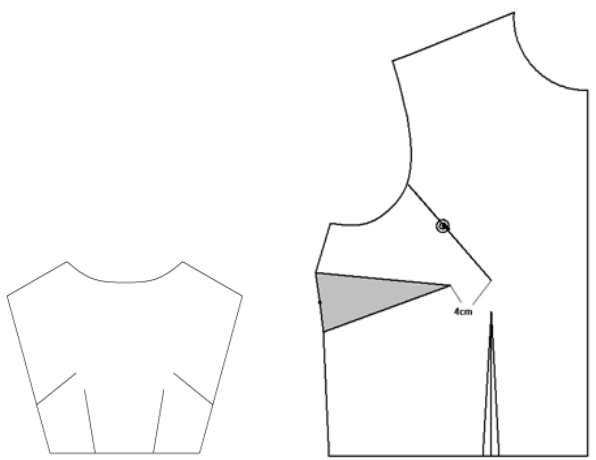

Fig. 3. Production Sketch and Pattern Transfer of Single Concentrated Armpit Dart
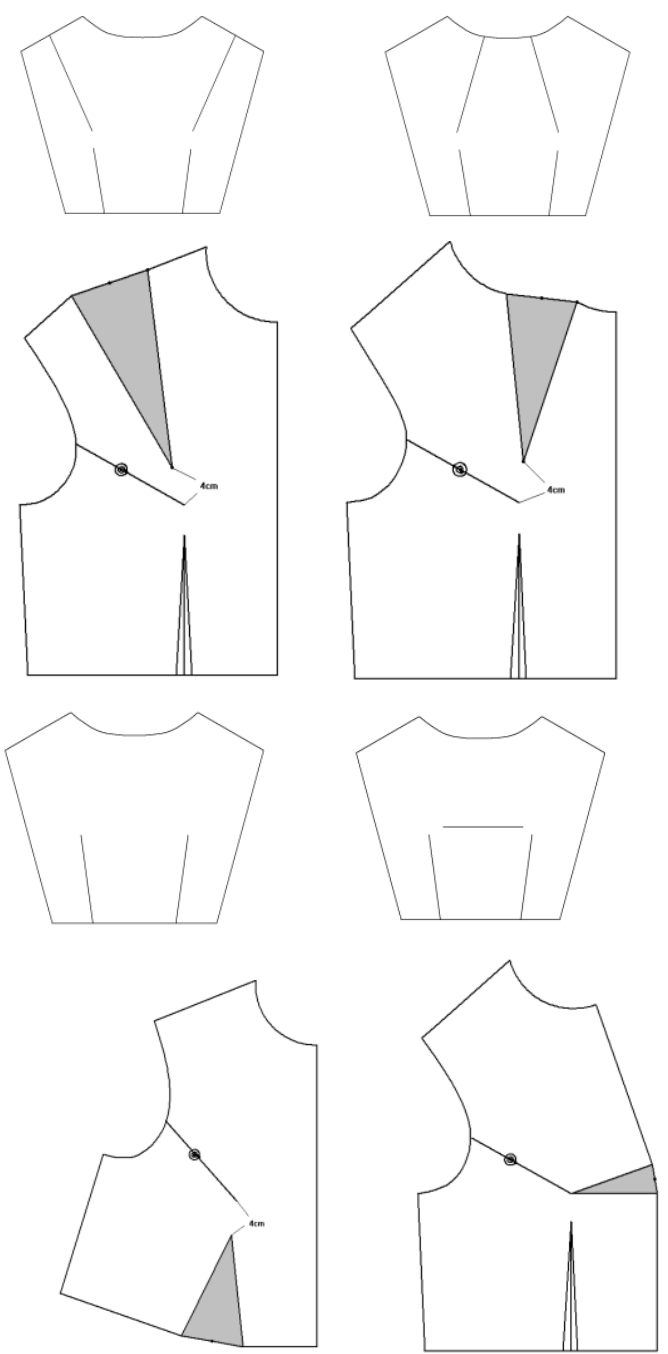

Fig. 4. Basic Transfers of Shoulder Dart, Collar Dart, Waist Dart and Seam Dart 
When centralized or decentralized use of dart is used, the transfer of dart is formed. The so-called dart transfer is the process changing its original location based on requirements of style design, or by virtue of other methods such as dividing lines, pleats to form a new three-dimensional modeling. It is the core of creative design of modern women's dress.

In clothing, each dart consists of three parts, namely, the location of dart, the angle of dart and the length of dart, which are known as the three properties of dart. Among which, the change of location of dart is related to the design of garment style. The dart that can be transferred shall meet one condition, namely, dart point shall aim at the convex point of human body, such as the bust point (BP point) and the shoulder blade point, or it cannot be transferred. In the transfer process of dart, the angle of dart (the size of the dart) will not change, to ensure the effect of shaping body surface will not change, but the length of dart will vary according to different locations of dart. Generally, the shorter the distance to human body convex point, the short the dart length, the longer the distance, the longer the dart length. If the dart point does not aim at the convex point of human body, then the margin can only be removed by dart eliminating.

The commonly used methods of dart transfer can be divided into two kinds: one is to cut the paper pattern at the location of new dart, fold the original dart, so that the cut position can open, the amount of opening is the amount of new dart. This method is called "cutting method". The dart transfer can be understood intuitively and vividly using this method and this method is applicable to complex straight line or curve and even the multi-dart transfer. But it must be done by destroying the paper pattern, which is not conducive to the preservation of paper pattern drawing process; the second is prototype rotation method, that is, to achieve dart transfer by pressing the convex point and rotating the prototype. This method is easy to operate, but it is too abstract. It will be more effective to understand in combination with the actual production of garment. Of course, these two methods can achieve the same effect. The expression forms after dart transfer can also be divided into seam and pleat, which have greatly enriched the style and profile design of women's dress.

\section{DisCussion OF CREATIVE STRUCTURAL DESIGN METHOD TAKING Bust DART As AN EXAMPlE}

The following text discusses methods for creative structural design of women's dress taking bust transfer as an example.

First is the basic transfer of bust dart. Figure 3 is production sketch and paper pattern of single concentrated armpit dart. Transfer the bust dart of prototype to new dart using the prototype of front piece of clothing, and by virtue of the aforementioned dart transfer method. Similarly, a variety of basic dart transfer can be formed according to different locations of darts, namely, collar dart, shoulder dart, waist dart, seam dart and so on (see Fig. 4). It can be seen from basic transfer that, dart design can be conducted around the bust point. Of course, we can also transfer bust dart into the design of multiple darts with unequal amount. Select the front piece of garment prototype, work out new dart line according to production sketch, and mark the auxiliary line to connect the dart end to bust point. Use the dart transfer methods, divide the total bust dart volume into 3 parts with unequal amount, and transfer them to 3 new darts, so that the total dart volume remains unchanged, and finally amend the shape of new dart at collar, ignoring the unnecessary dart volume (Fig. 5).

Second, form the seam design using the combination of bust dart and waist dart. The so-called seam is to transfer onepiece structure to the combination structure with two or more pieces using dart cutting, in order to achieve the purpose of reasonable structure and style design. For example, the classic princess line structure, Pineal line structure, etc. (Fig. 6). We can also arbitrarily connect bust dart and waist dart into numerous kinds of seam design using this method. Connect collar dart and waist dart, seam dart and waist dart and so on (Fig. 7).

Third, combine dart transfer with the pleats. The combination of the two can form seam design which is decorative and slim. Previously, pleat design is often used in formal dress design. But with the ready-to-wear trend of formal dress elements, forming pleats from dart transfer has been a common method of structural design (Fig. 8).

Fourth, combine dart transfer with decorative cutting line to achieve a new design effect. Stitches can be divided into decorative stitch and functional stitch according to the modeling purpose in clothing. Among which, the stitch with function of dart transfer is called functional stitch, otherwise is the decorative stitch. For example, the combination design of dart and shoulder yoke has perfectly shaped human shoulder structure while enriching visual effects (Fig. 9).

Fifth, combine dart transfer with pleats and decorative cutting line. Such combination of multiple elements is an indepth understanding of structural design, which can create a richer visual effect (Fig. 10).

Sixth, combine dart transfer with some independent threedimensional modeling. The dart transfer itself is to shape three-dimensional form. When it is combined with some of the local three-dimensional modeling, there will be enlightenment on unique visual aesthetic and design thinking (Fig.11).

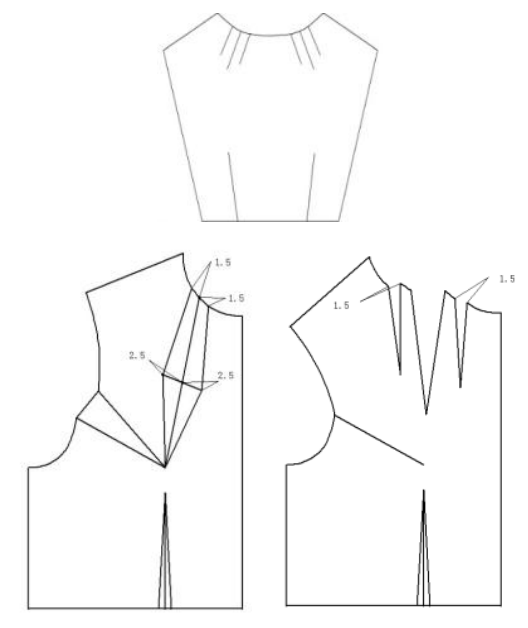

Fig. 5. Transfer Bust Dart into the Design of Multiple Darts with Unequal Volume 

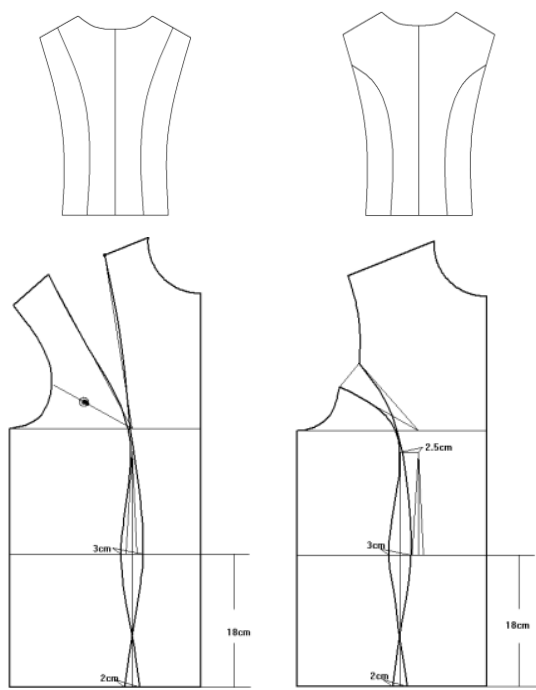

Fig. 6. Pattern Design of Classic Princess Line Structure and Pineal Line Structure
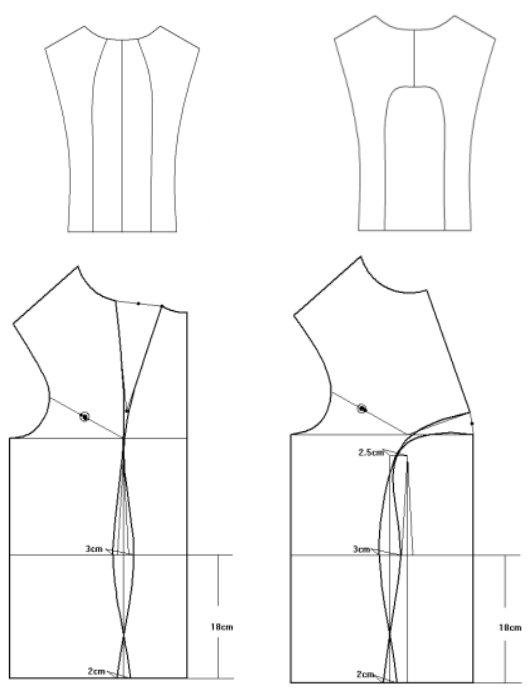

Fig. 7. Pattern Design of Connecting Collar Dart and Waist Dart, Seam Dart and Waist Dart

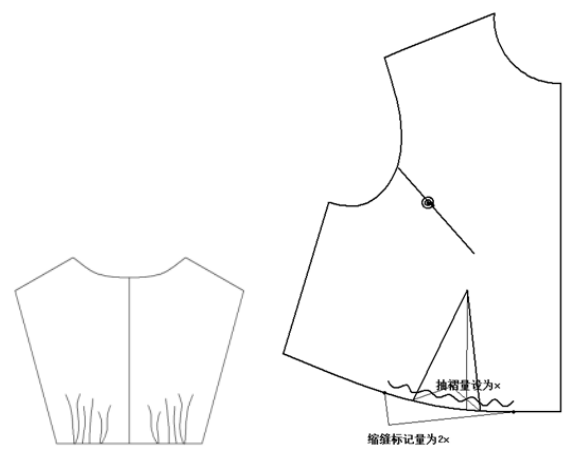

Fig. 8. Pattern Design of Dart Transfer to form Pleats

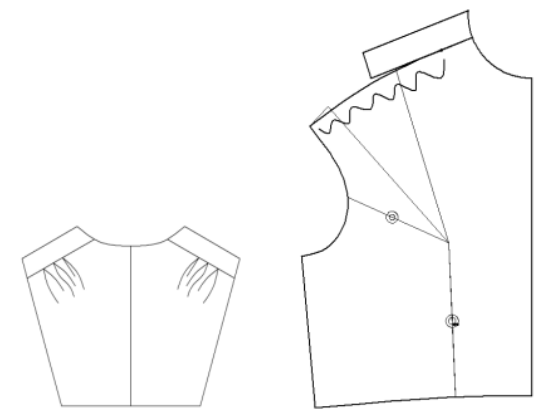

Fig. 9. Pattern Design of Combination of Dart Transfer and Decorative Cutting Line

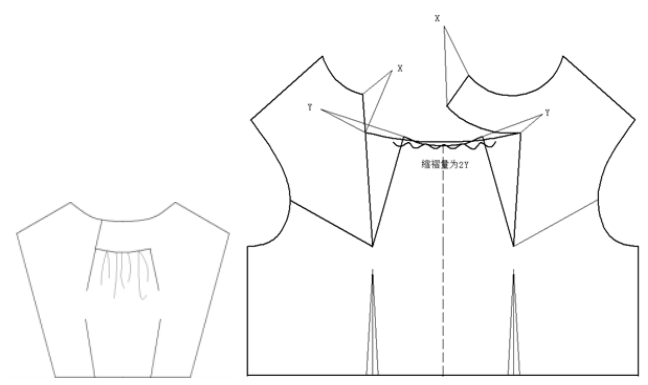

Fig. 10. Pattern Design of Combination of Dart Transfer with Pleats and Decorative Cutting Line
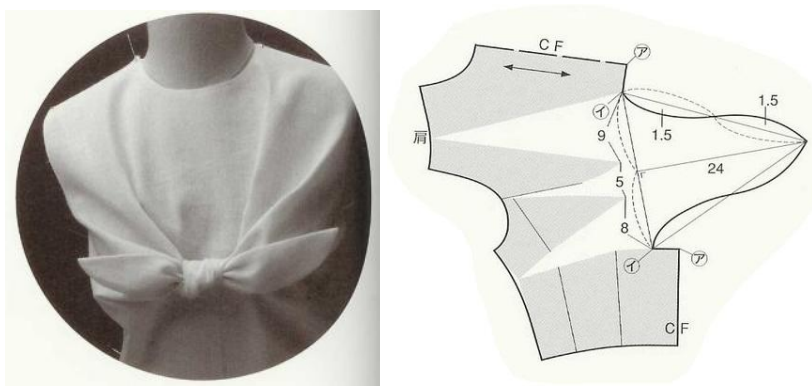

Fig. 11. Pattern Design of Combination of Dart Transfer with some Independent Three-dimensional Modeling (Works of Nakamichi Tomoko)

\section{CONCLUSION}

All in all, the garment creative design has gradually extended from simple style design to every aspect of garment processing, including fabric design, pattern design, structural design and technological design. Different from the previous visual design, starting from the internal structure of garment to explore more design space and aesthetic taste will bring a brand new space effect and unique design charm to the garment, which also constantly inspires and prompts designers to continue to explore structural design.

\section{REFERENCES}

[1] Nakamichi Tomoko. Magic Pattern, Bunka Publishing Bureau, 2005

[2] Miyoshi Tomoko. Clothing Model Theory: Theoretical Articles, China Textile Press, 2006

[3] Wang Jianping. Structural Design of Women's Dress, Donghua University Press, 2014 
[4] Liu Ruipu. TPO Branded Women's Dress Design and Plate-making Training, 2010 